\title{
ASSOCIATION OF CARIBBEAN UNIVERSITIES AND RESEARCH INSTITUTES (UNICA)
}

P.O. Box 11532

Caparra Hights Station

San Juan

Puerto Rico 00922

Tel: (809) 720-4381

Fax: (809) 567-9219

President: Dr. René Acheen

Secretary-General: Dr. Thomas Mathews

A non-profit, non-governmental, educational organization authorized to operate in Jamaica, Venezuela, Colombia, Puerto Rico and Dominican Republic. Its aims are to foment cooperation between institutions of higher education operating in the Carribean, particularly in the fields of agricultural, science, environmental, educational studies, etc

.Principal publications: UNICOM (newsletter, quarterly); Caribbean Educational Bulletin (suspended).

L'Association des universités et instituts de 1967 recherche des Caraibes est une organisation non-gouvernementale du domaine de l'éducation, à but non lucratif, dont le fonctionnement est autorisé en Jamaïque, au Venezuela, en Colombie, à Puerto Rico et en République dominicaine. Les objectifs de l'Association sont de favoriser la coopération entre institutions de l'enseignement supérieur fonctionnant aux Caraïbes, spécialement dans les domaines de l'agriculture, des sciences, de l'environnement et de l'éducation.

Parmi les publications principales: "Unicom" (bulletin trimestriel); "Caribbean Educational Bulletin" (suspendu). 